\title{
IPSA Scientia
}

Artículo de Investigación

\section{Competencias pedagógicas y tecnológicas del docente para el diseño instruccional en educación virtual universitaria Pedagogical and technological competences of the teacher for instructional design in university virtual education}

\author{
DBUITRAGO-BOHÓRQUEZ, Blanca \\ Universidad Bicentenaria de Aragua, San Cristóbal, Venezuela \\ ๑SÁNCHEZ, Haydeé \\ Universidad Nacional Abierta, San Cristóbal, Venezuela \\ Autor corresponsal: blancaemmabuitrago@gmail.com
}

Recibido: 03-02-2021; Aceptado: 25-06-2021; En línea: 28-06-2021

DOI: https://doi.org/10.25214/27114406.1054

Cómo citar este artículo:

Buitrago-Bohórquez, B. \& Sánchez, H. (2021). Competencias pedagógicas y tecnológicas del docente para el diseño instruccional en educación virtual universitaria. IPSA Scientia, revista científica multidisciplinaria, 6(2), 82-100. https://doi.org/10.25214/27114406.1054

Resumen - El objetivo de la investigación fue construir las competencias pedagógicas y tecnológicas en los docentes universitarios para el diseño instruccional en educación virtual de la Universidad Bicentenaria de Aragua, Venezuela. A partir de la experiencia, se pudo constatar que este requerimiento exige preparación tanto pedagógica como tecnológica para una educación de calidad frente a la realidad post pandemia. El abordaje del objeto de estudio asume el método inductivo propio de la investigación cualitativa, con apoyo en el método etnográfico y el hermenéutico dialéctico, Los actores sociales en esta investigación son, docentes y expertos a través de entrevistas en profundidad y la observación participante, fueron fuentes de información. Los resultados ofrecieron aristas austeras para la definición de competencias conformadas por: el saber hacer del docente y la infraestructura tecnológica, propios de las competencias tecnológicas, por una parte, y por la otra, el saber del docente, propio de las competencias pedagógicas. Se concluye que las competencias requeridas para los procesos de diseño instruccional en la educación virtual, se sustentan en conocimientos, actitudes, habilidades y destrezas expresadas en el ser, conocer, hacer y convivir del docente para orientar su práctica virtual, mediante un diseño de la instrucción que se fundamente en teorías relativas al aprendizaje humano para conducir al estudiante a desenvolverse en diferentes contextos a partir de mediación pedagógica con las tecnologías educativas que lo llevará a probarse, evaluarse y revisarse de forma efectiva y continua, es decir al recibir atención de manera satisfactoria a sus necesidades de aprendizaje en ambientes virtuales.

Palabras clave: Competencias pedagógicas, competencias tecnológicas, diseño instruccional, docente universitario, educación virtual 


\begin{abstract}
The objective of this research was to build the pedagogical and technological competencies needed thought the university teachers for the instructional design in virtual education of the Bicentennial University of Aragua, Venezuela. Based on experience, the researchers were able to verify that this requirement requires both pedagogical and technological preparation for quality education in the face of post-pandemic reality. The approach to the object of study assumes the inductive method of qualitative research, supported by the ethnographic method and the dialectical hermeneutical method. The social actors in this research are teachers and experts through in-depth interviews and participant observation, they were sources of information. The results offered austere edges for the definition of competences made up of: the know-how of the teacher and the technological infrastructure, typical of technological competences, on the one hand, and, on the other, the knowledge of the teacher, typical of pedagogical competences. It is concluded that the competencies required for the instructional design processes in virtual education are based on knowledge, attitudes, abilities and skills expressed in the being, knowing, doing and living together of the teacher to guide their virtual practice, through a design of the instruction that is based on theories related to human learning to lead the student to develop in different contexts from pedagogical mediation with educational technologies that will lead him to be tested, evaluated and reviewed effectively and continuously, that is, by receiving attention in a way satisfactory to your learning needs in virtual environments.
\end{abstract}

Keywords: Pedagogical skills, technological skills, instructional design, university teacher. virtual education.

\title{
Introducción
}

La llegada del siglo XXI ha estado marcada por la aparición de innovaciones en todos los ámbitos de la existencia, entre los cuales se encuentra la educación. Uno de los graves problemas que ha aquejado al sector educativo en cuanto al proceso de enseñanza en las diversas modalidades, lo constituye sin duda la falta de competencias de los docentes para enfrentar el reto de la educación virtual. Este fenómeno influye en la formación de los estudiantes que buscan una nueva alternativa en este tipo de educación por considerarla más accesible y flexible para su régimen cotidiano de vida. Al respecto, Roblizo \& Cozar (2015) han dicho: "actualmente concebir la vida en las aulas sin la presencia de las TIC es algo impensable” (p. 24). Por ello, la educación enfrenta numerosos desafíos debido a los cambios que involucran nuevas modalidades de estudio, procedimientos, metodologías, recursos, ambientes, estrategias, en fin un sinnúmero de aspectos que contribuyen a la integración de la tecnología en los procesos de enseñanzaaprendizaje como parte de la cultura innovativa institucional (Cohen-Granados et al., 2020).

Al ofrecer programas de educación en todos los niveles en la modalidad virtual, se hace necesario contar con personal docente que posea las competencias pedagógicas y tecnológicas acordes a la exigencia del diseño instruccional requerido para el desarrollo de programas en ambientes virtuales de aprendizaje. Esta necesidad se acrecienta en estos tiempos de pandemia donde las instituciones han tenido que adaptarse a los requerimientos de las clases virtuales en todos los niveles de la educación, lo cual requiere que el docente se forme, se actualice y adquiera las competencias requeridas para diseñar la instrucción. Roblizo \& Cozar (2015), otorgan importancia a los cambios en educación dados en todos los niveles, pues siempre se introducen mejoras en los procesos de gestión educativa, los cuales incorporan cada día, materiales y recursos didácticos mediados por la tecnología, materiales multimedia interactivos que junto a nuevas metodologías de enseñanza, permiten el desarrollo de habilidades cognitivas, así como la adquisición y consolidación de las competencias que exige la educación virtual. Esta 
última, está siendo altamente demandada por la sociedad para el proceso de formación profesional, porque ofrece nuevas alternativas de comunicación entre el docente y los estudiantes, los estudiantes y los materiales.

En atención a lo expuesto, el Instituto Internacional de Planeamiento y Educación (IIPE), organismo adscrito a la Organización de las Naciones Unidas para la Educación, la Ciencia y la Cultura (UNESCO); con sede en Paris, Francia ha planteado la necesidad de construir proyectos educativos, donde se debe estudiar su viabilidad, por cuanto resulta necesario manejar tres condiciones: el saber hacer, el querer hacer y el poder hacer. Para ello, es preciso conocer, tanto el recurso humano como tecnológico con el que cuenta una institución educativa y esto es posible mediante la realización de un estudio donde se resalten los aspectos que requieren los docentes virtuales, para desarrollar las competencias pedagógicas y tecnológicas. Para ello:

El Instituto está comprometido a ayudar a los Estados Miembros a alcanzar sus objetivos educativos tanto para las generaciones actuales como para las futuras. Si el mundo parece estar cambiando a una velocidad cada vez mayor, el IIPE cambia con él, manteniendo a la vez sus principios fundacionales. El IIPE tiene la esperanza de que con su apoyo, los países sigan teniendo éxito en la planificación de un futuro mejor, con oportunidades educativas inclusivas y equitativas para todos (UNESCO-IIPE, 2018, p. 4)

Al contar con ese apoyo y ante el fin afrontar los grandes desafíos, las universidades podrán asumir acciones en este nuevo escenario; para ello se requiere de proyectos cuyo objetivo se centre en analizar y promover las competencias de los docentes hacia nuevas perspectivas pedagógicas y tecnológicas con el fin de contribuir a la enseñanza y facilitación del aprendizaje virtual. Esta situación, hace eco en tiempos donde la tecnología presenta la opción que tienen los estudiantes, para tener acceso a la educación a distancia por motivos de distanciamiento social; entonces, se requiere de docentes competentes que estén dispuestos a asumir la nueva realidad. En este sentido, la oportunidad es propicia para considerar temas que atañen al común de los docentes en todos los niveles educativos, quienes tienen en sus manos el compromiso de rediseñar la función docente a través de buenas prácticas (De-Vincenzi, Marcano \& Macri, 2020) desde un entorno virtual.

$\mathrm{Al}$ abordar el tema de la educación virtual en los procesos de enseñanza y aprendizaje, ésta tiene lugar parcial o totalmente a través de la red y los recursos digitales de enseñanza y aprendizaje. Gamarra (2021), considera que "las expectativas de generar innovación en la mente de cada estudiante están en la mano de un docente, la tarea de adaptarse y tomar compromisos para asumir retos en esta realidad cambiante sin perder esa función formativa” (p. 13). De manera que, la educación enfrenta desafíos debido a los cambios que involucran las nuevas modalidades educativas, procedimientos, metodologías, recursos, ambientes, estrategias didácticas. Es decir, aspectos que exigen la integración de las tecnologías en el proceso de enseñanza y aprendizaje, dada la importancia que involucran las ayudas digitales, el quehacer docente profesional, las formas o maneras de enseñar y las competencias docentes (pedagógicas y tecnológicas). Todo ello, implica realizar modificaciones en cuanto al modo de establecer la comunicación en entornos virtuales; así como aprovechar las potencialidades que ofrece la red en materia educativa. De ahí, la necesidad de formar estudiantes de manera integrada y articulada con los diseños instruccionales y la institución, para lo cual es recomendable: recurrir a buenas fuentes de 
Competencias pedagógicas y tecnológicas del docente para el diseño instruccional en educación virtual universitaria

información, producir contenidos de calidad, clasificar los materiales de acuerdo a las áreas de estudio e interactuar con otras personas expertas en el área para conectar el conocimiento.

Ante este panorama, son muchos los estudios referidos a las competencias docentes y el diseño instruccional, que han dejado claro la necesidad de abordar el tema desde diferentes ópticas. Un primer ejemplo, lo constituye el estudio realizado por Jiménez (2015), sobre los estándares TIC en educación en los futuros docentes de la Facultad de Educación de la Universidad Complutense de Madrid; el mismo hace énfasis en la revolución que han ocasionado las TIC en todas las áreas de la sociedad, a la que se le ha denominado la Sociedad de la Información y del Conocimiento. Frente a esta revolución, las competencias que esta sociedad digital exige a sus profesionales de la educación, requieren de una formación inicial sólida con dominio de los estándares TIC para educación, que la misma sea continua y de calidad.

Para Jiménez (2015) el docente deberá buscar, seleccionar y organizar las actividades que propondrá al estudiante, haciendo que esta nueva función descentre del acto didáctico para garantizar la adquisición de las competencias trazadas. Todo esto, será más factible, ameno y mejor si el educador posee las competencias TIC en educación. Con base en estas ideas, la pertinencia del estudio radica en lo significativo de requerir docentes competentes en TIC contemplando sus estándares para el desempeño en la docencia universitaria, aunado a la formación en diseño instruccional para enfrentar el desafío que implica ser docente virtual exitoso y contribuir a la formación integral del estudiante bajo esta modalidad.

Otra experiencia importante, es el aporte de Carrillo \& Roa (2019), en la Universidad de la Sabana con la creación de un prototipo de curso virtual alojado en el sistema de gestión de aprendizaje Moodle, el cual tuvo en cuenta los criterios de diseño instruccional según el modelo ADDIE, para fomentar entre profesores y facilitadores la apropiación de elementos teóricos y prácticos de estrategias instruccionales de la segunda fase de este modelo: el diseño. Los citados autores hacen énfasis en los grandes desafíos de la educación virtual para crear ambientes de aprendizaje más reales, con mayor interacción entre estudiantes, y entre estos y los profesores, la cual debe ser de calidad, sostenidos dentro de un marco teórico de la psicología educativa.

Esta investigación dejó aprendizajes significativos sobre el diseño ADDIE y la educación virtual; que responden a espacios virtuales de aprendizaje obtenidos a través del diseño instruccional, donde se explica, cómo desarrollar estos ambientes mediante estrategias instruccionales que tomen en cuenta los procesos cognitivos de aprendizaje.Su significatividad, estriba en que, no se debe tomar a la ligera este desarrollo y prescindir de un modelo o guía para su implementación, $\mathrm{Al}$ efecto Carrillo \& Roa (2019) sugieren:

(...) es necesario formalizar en todos los escenarios tanto en el de educación formal como informal, los lineamientos necesarios para el diseño de programas instruccionales efectivos, de calidad, atractivos, innovadores y sobre todo que tengan en cuenta los procesos de aprendizaje en las personas. Lo anterior invita a continuar desarrollando el prototipo instruccional del modelo ADDIE en su totalidad, en virtud de responder a los desafíos y retos de la educación virtual hoy en día (p. 73). 
Por último, resulta relevante el estudio de Aliendres (2012), sobre competencias del docente para el logro de una educación de calidad en el Instituto Universitario de Tecnología de Puerto Cabello, Venezuela. El propósito de la investigación fue analizar las competencias del docente para el logro de una educación de calidad. Esta investigación enfatiza en que un porcentaje moderado de los docentes exhiben las competencias básicas, genéricas y específicas, por lo que se recomienda crear programas y cursos de actualización con el fin de que los docentes desarrollen al máximo sus competencias para el logro de la calidad educativa que requiere la institución y el país. Es un hecho la preocupación de los investigadores por las competencias docentes para enfrentar las nuevas demandas de la educación en la sociedad actual, situación que sigue vigente y amerita atención a través de la investigación.

\section{Importancia de la investigación en la actualidad}

Vale resaltar la notabilidad que ofrecen los estudios anteriores al tema de investigación, en particular lo que atañe al proceso de diseño instruccional en la educación virtual y las competencias docentes. De ahí que se haga necesario llevar el estudio a la actualidad desde la perspectiva del panorama que se vive en la educación por efecto de la pandemia y cómo esta afecta el hecho educativo y sus actores sociales, docentes y estudiantes.

En este escenario, la educación virtual ha adquirido un valor sin precedentes que marcará un antes y un después en la práctica docente, lo cual se ha evidenciado en la crisis de pandemia (COVID-19) que vive la humanidad. Es notable cada día mayores exigencias para conseguir un empleo, por lo que no escapa el hecho de que los estudiantes se ven en la necesidad de optar por incursionar en la virtualidad para sus estudios de grado y postgrado, ellos enfrentan retos al buscar la mejor opción de formación profesional. Para ello, es preciso que las universidades cuenten con aliados clave y que mejor que tener en sus haberes, docentes virtuales con competencias en el área tanto pedagógica como tecnológica, los cuales estén prestos a trabajar en entornos virtuales sin ninguna limitación aptitudinal.

En atención a lo expuesto en el párrafo anterior, Russi (2020), recientemente ha señalado: “En esta situación marcada por el miedo y la incertidumbre frente a los acontecimientos sanitarios y sus consecuencias sociales, laborales y económicas, la figura del docente ha cumplido un rol fundamental que ha ido mucho más allá de los aspectos pedagógicos” (p. 3). Realidad que lleva a una transformación de la enseñanza presencial a la enseñanza dirigida desde lo remoto, donde optar por la virtualidad es la opción más flexible y cómoda para acatar las normas de bioseguridad que imponen los organismos competentes. En efecto, la situación pandémica trajo desafíos a la educación virtual y surgieron más posibilidades para la formación continua del docente con apoyo en los recursos tecnológicos y materiales de la web, lo cual favorece el desarrollo profesional en la modalidad virtual.

Ante el panorama descrito, se sugiere que los docentes desarrollen competencias pedagógicas y tecnológicas, para enfrentar el desafío que exige la educación virtual en el nivel superior, lo es también el cambio de paradigma propiciado por el uso de las tecnologías en la dinámica profesor activo versus estudiante pasivo (Díaz \& Serra, 2020), para pasar a un nuevo modelo donde todos los actores académicos interactúan en la definición del contenido y una enseñanza acorde a los preceptos del paradigma constructivista actual, lo cual justifica el aporte práctico del presente trabajo. Es importante diseñar las competencias pedagógicas y tecnológicas que requiere el 
Competencias pedagógicas y tecnológicas del docente para el diseño instruccional en educación virtual universitaria

docente antes de enfrentar su práctica educativa en ambientes virtuales, pues la misma en algunas ocasiones conduce al docente tradicional a un rol de docente virtual sin tener idea del uso de las tecnologías en la educación virtual de cualquier institución educativa.

La importancia de esta investigación también está dada, porque los resultados son un referente educativo innovador para que la Universidad Bicentenaria de Aragua de Venezuela, y otras instituciones a nivel nacional e internacional, puedan visualizar la necesidad e intereses académicos de los actores sociales, en este caso los docentes de grado y postgrado y se aspira que la misma pueda contribuir con el desarrollo de las competencias pedagógicas y tecnológicas necesarias para los procesos de diseño instruccional del docente virtual. Además, las competencias descritas, en el marco de la educación virtual, permite la articulación dialéctica de la formación virtual de los estudiantes, a partir de las necesidades de formación.

Desde el punto de vista institucional, esta investigación permitirá el mejoramiento de las relaciones con el entorno social y en efecto un cambio importante en la calidad académica en todos los niveles de la educación mediante el uso y aplicación de la tecnología, para generar los cambios aptitudinales requeridos en esta modalidad virtual. Asimismo, el aporte práctico está dado por el cambio en la aptitud del docente, la cual aún se caracteriza por una didáctica tradicional con escaso uso de los recursos y herramientas que proporciona la web para a educación virtual. El diseño por competencias favorece en los docentes el desarrollo de habilidades y destrezas, mientras enseñan con trascendencia pedagógica y tecnológica, así como ética, educativa, institucional y legal. Tales conceptos atienden a una educación de calidad que al ser realizada con competencias contribuirá a una actuación del docente desde su formación con honestidad, además la credibilidad que el estudiante tenga de la institución al ofrecer programas de grado y postgrado que estén lícitamente aprobados por el Ministerio de Educación y que cumplan los estándares de calidad que este exige.

Las metas institucionales apuntan al mejoramiento de la calidad en los desempeños de los estudiantes de postgrado en el caso específico de esta investigación, desde el punto de vista de los procesos de aprendizaje, lo cual justificó generar elementos estructurales que permitieron la creación de las competencias pedagógicas y tecnológicas en los procesos de diseño instruccional de los aprendizajes. Por ello, la trascendencia de la investigación se refleja en los beneficios para los estudiantes de postgrado y grado, porque al ofrecer alternativas innovadoras para su desempeño en la virtualidad, elevará su motivación y vocación por los programas de postgrado en educación y otras disciplinas que ofrece la UBA.

\section{Conceptos Fundamentales}

Para el desarrollo de competencias pedagógicas se requiere de la intervención de agentes que contribuyan de manera pertinente a la realización de procesos en beneficio de tal propósito; así pues, las prácticas pedagógicas vistas como el proceso integrado de actividades que cumple el docente mediante una didáctica definida, debe incidir favorablemente en otras competencias, como el caso de las tecnológicas. En relación con las competencias del docente, el Observatorio de Innovación Educativa (2015) señala: 
Una competencia es la integración de conocimientos, habilidades, actitudes y valores que permite a una persona desenvolverse de manera eficaz en diversos contextos y desempeñar adecuadamente una función, actividad o tarea. Las competencias facilitan el desarrollo de una educación integral ya que engloban todas las dimensiones del ser humano: saber, saber hacer, y saber ser y estar” (p. 6).

El informe enfatiza en la necesidad de definir el nivel de conocimientos y habilidades que pueda alcanzar un estudiante al final del proceso educativo, como resultado de participar en el desempeño de su profesor para adquirir sus propias habilidades. En otras palabras, la competencia del docente contribuye a la competencia del estudiante.

En la última década, las competencias se han constituido en el eje articulador del sistema educativo, por ejemplo, en Colombia, el Ministerio de Educación Nacional de Colombia, MEN (2013) define competencia como: “el conjunto de conocimientos, habilidades, actitudes, comprensiones y disposiciones cognitivas, socioafectivas y psicomotoras apropiadamente relacionadas entre sí para facilitar el desempeño flexible, eficaz y con sentido de una actividad en contextos relativamente nuevos y retadores” (p. 31). El propósito que esta entidad plantea, coincide con las tendencias mundiales de la educación para el siglo XXI y con las recomendaciones de expertos que aun cuando no siempre coinciden en las competencias específicas a desarrollar, sí están de acuerdo en que para prepararse para la sociedad del conocimiento se necesita la flexibilidad que ofrece el enfoque por competencias tanto en los procesos de formación como en los sistemas de evaluación. Todo esto irá de la mano con la voluntad que tenga tanto la institución formadora, como el docente en acatar los propósitos del MEN, ya que a juicio de las autoras de este artículo, cada modalidad educativa requiere sus propias competencias las cuales son indispensables en la formación de calidad.

En lo referente a mejorar la calidad de la educación en todos los niveles, el MEN (2013) ha dicho:

(...) se considera el desarrollo profesional de los docentes como uno de los principales factores que aportan al fortalecimiento de la calidad educativa y por ende se hace relevante una formación que le permita cualificar sus prácticas pedagógicas, al tiempo que se desarrolla como persona y como profesional, para responder a las expectativas, necesidades y demandas de la sociedad y contribuir desde su quehacer al proyecto educativo de Nación. Por esto, el Ministerio apoya, orienta y dinamiza la formación de los docentes para que amplíen sus conocimientos, propongan estrategias didácticas, metodologías flexibles y modelos pedagógicos innovadores que garanticen a sus estudiantes independientemente de su zona (rural o urbana), sector (oficial o privado) u otras condiciones particulares, la participación en una educación, pertinente y de calidad (p. 15).

Allí se observa, cómo en Colombia se ha concebido la necesidad de crear y hacer seguimiento a los programas de desarrollo profesional y además sugieren hacerlo con la participación activa de los docentes y directivos docentes en coordinación con las Secretarías de Educación y de esta manera poder determinar en qué nivel se encuentra los docentes antes y después de cada formación. En este sentido el MEN (2013) ha establecido: "Las Secretarías de Educación Departamentales y Distritales, (...) organizarán programas dirigidos a fomentar estudios 
científicos de la educación, con el objeto de fortalecer la formación personal y profesional de los educadores que prestan el servicio en su territorio" (p. 24). Estos programas, son un referente para la educación venezolana, pues la necesidad de contar con docentes con competencias, lo cual se desprende de la presente investigación, conlleva a una articulación de políticas educativas implementadas en otros países.

Ahora bien, retomando la educación virtual, existen importantes tendencias de innovación dentro de esta modalidad relacionadas con el diseño instruccional. Al respecto, García Aretio (2020), señaló: "es una constante en mis afanes sobre estos temas, el preservar la concordancia, coherencia e interrelación entre todos los elementos del diseño, al igual que entre todas las partes o componentes...” (p. 9). En acuerdo con el autor, el diseño instruccional es el elemento de impacto que debe llenar las expectativas de los estudiantes mediante un trabajo coherente e interrelacionado del docente con competencias pedagógicas y tecnológicas acordes a las exigencias del currículo y el apoyo de la institución.

De manera que, el diseño instruccional involucra un todo coherente, una realidad social para un sistema educativo en cualquier modalidad que ofrezca formación profesional para llenar las expectativas de quienes aprenden. Utilizar un modelo de diseño instruccional facilita la elaboración del material por parte de los involucrados en la producción los mismos, también facilita la gestión del proceso a los profesores y la ejecución de este a los estudiantes mediante la educación virtual.

La educación virtual, según Sánchez (2020), "presenta su evolución y sus respectivos aportes para el reconocimiento del estudiante competente, que se ajusta a un modelo estándar y que describe sus habilidades básicas” (p. 18). Esta realidad requiere de docentes competentes para afrontar los retos que ello implica; es decir, satisfacer las necesidades del estudiante sin estar presente físicamente siempre y sin que se note ese vacío o sensación de ausencia. Entonces, la educación virtual requiere de ambientes que se constituyan en espacios mediadores entre los estudiantes, los materiales educativos y las actividades docentes y formativas. Para ello, los docentes deberán contar con las competencias necesarias a los fines de planificar las actividades académicas y preparar los materiales didácticos mediante un diseño instruccional cónsono con el programa a impartir en la virtualidad.

Al considerar la educación virtual mediante Espacios Abiertos de Aprendizaje (EAA), Calderón (2020), ofrece un aporte interesante al proponer que "la virtualización de un aprendizaje no solo es el desarrollo de material didáctico llamativo, sino que conlleva emplear una estrategia de diseño curricular y una fuerte base epistemológica que orienten todo el resto del proceso" (p. 100). La citada autora advierte, que lo virtual ya no es un asunto de especialistas, ni de correo electrónico o el llamado ciberespacio, pues es básico contar tanto con las aptitudes y actitudes del docente, así como otros elementos necesarios, para la creación de espacios virtuales de aprendizaje. Estos espacios están relacionados con los recursos tecnológicos, (videos tutoriales, mapas conceptuales, foros de debate, podcast y las presentaciones en diferentes estilos y modalidades) y los medios y herramientas (entornos virtuales).

En relación con el enfoque teórico de la Educación Virtual, se han considerado el Conectivismo, el Constructivismo, y Humanismo. Se hizo un recorrido por estas tres teorías, las cuales 
sustentaron por una parte el diseño instruccional y por otro la educación virtual. Las mismas vienen a reafirmar la evolución de la educación virtual y el cambio de una educación pasiva a una más activa construida por el propio estudiante con la motivación que ejercen las TIC con un tutor competente, con habilidades y destrezas pedagógicas y tecnológicas.

En primer lugar, se tiene la concepción de una nueva teoría denominada Conectivismo. Al referirse a las ecologías del aprendizaje, Siemens (2010), consideró que estas "son entornos de conocimiento compartido, fomentando las conexiones y fuentes de conocimiento, dando lugar a la circulación de éste. Son libres, inarticuladas, dinámicas, adaptables, confusas y caóticas. Por eso habla de un organismo vivo” (p. 10).

Por otra parte, al analizar diferentes concepciones que se han generado frente al Constructivismo, en términos generales, es una interpretación de la realidad, una opción epistemológica particularmente interesante para la Psicología porque permite abordar de un modo creíble y sugerente la evolución del ser humano. Es una forma de adquisición del conocimiento que puede inspirar la formulación de modelos y teorías, o para reflexionar métodos de investigación; pero no puede reducirse a una teoría o a una metodología concreta. Tampoco es un modelo educativo, ni prescribe un determinado modo de enseñar, aunque las prácticas educativas suelen estar ligadas a un conjunto de decisiones que pueden basarse en una concepción constructivista de lo que es enseñar y aprender.

Este enfoque parte del supuesto, de que el estudiante es un ser activo que recibe y procesa información e interactúa con el objeto de estudio para edificar su propio conocimiento de acuerdo con su entorno; de igual manera se infiere la manera como se asume el proceso de conocer: una construcción mental claramente subjetiva e idiosincrática. Al respecto, Álvarez de Zayas \& González, citados en Prada (2010), expresan:

Cuando el proceso formativo se desarrolla y se construye de un modo sistémico se está en la presencia del proceso didáctico, el cual posee un fundamento teórico dirigido a la educación, desarrollo e instrucción de los estudiantes, proceso tutelado por un personal especializado (p. 123).

Ese proceso formativo del que hablan conduce a inferir que el constructivismo es sin duda alguna uno de los principales fundamentos teóricos de la instrucción y por ende del diseño instruccional. Esta concepción constructivista se organiza en torno a ideas que conducen a pensar que el estudiante es el responsable último de su propio aprendizaje, y su actividad mental constructiva se aplica a contenidos que poseen ya un grado considerable de elaboración, lo que quiere decir que el estudiante no tiene que inventar o descubrir todo el conocimiento escolar. En este caso, la función del docente virtual no se limitaría a crear condiciones óptimas para que el estudiante despliegue una actividad mental constructiva, sino que pudiera guiar explícita y deliberadamente dicha actividad, la cual consiste en enlazar los procesos de construcción del estudiante con el saber colectivo culturalmente organizado por este. Es decir, el docente pudiera realizar el diagnóstico de clase inicial para conocer el colectivo de estudiantes para orientar el proceso didáctico mediante un diseño ajustado a sus necesidades.

Por último, el Diseño Instruccional para la educación virtual concibe el humanismo, la psicología cognitiva, el constructivismo, la teoría cognitiva de Piaget, la teoría del procesamiento 
de la información y en el rescate del liderazgo del docente. En lo pedagógico este modelo critica la enseñanza entendida como simple trasmisión de conocimientos mediante adquisición de competencias, para encaminarla como proceso constructivo apoyado en las experiencias, conocimientos y actitudes previas encauzadas al logro de aprendizaje significativos, mediante métodos comunicativos, con la vida, resolución de problemas y la formación integral de la persona.

\section{Materiales y Métodos}

Esta investigación asumió el enfoque cualitativo, mediante una investigación etnográfica con apoyo en la hermenéutica dialéctica. Se escoge la investigación etnográfica por cuanto permite la reconstrucción de escenarios culturales mediante análisis e interpretación de la información expresada por los investigados. La investigadora como parte del personal docente, con sus propias vivencias y en contacto con compañeros docentes, buscó descubrir las competencias en la práctica docente. En cuanto al método etnográfico, hermenéutico dialéctico, Flores \& Tobón. (2003) señalan que "la investigación educativa, se centra cada vez más en estudiar unidades sociales de dimensiones reducidas que permiten una observación directa. Tales unidades pueden ser grupos como bandas juveniles, barras u organizaciones como establecimientos educativos o grupos de clase” (p. 142).

Para el caso de la presente investigación, atendiendo a las ideas los citados autores, se contaba con el grupo de actores del proceso educativo en la virtualidad de la Universidad Bicentenaria de Aragua (UBA), quienes facilitaban programas acreditables al Doctorado en Ciencias de la Educación y la Maestría en Gerencia Educativa en los periodos correspondientes al 2017-2018, los cuales para el momento de la investigación estaba integrado por 18 docentes de postgrado de los que se seleccionaron 4 informantes clave ajustados a criterios particulares. Para la recolección de la información, se eligieron 2 docentes de doctorado en educación de la UBA, 2 docentes de maestría en educación de la UBA y 2 expertos externos en el área de diseño instruccional para ambientes virtuales como informantes clave. Los sujetos involucrados suman 6 en total.

La selección se realizó a partir de los siguientes criterios: (1) docentes contratados con amplia trayectoria en docencia universitaria en la modalidad presencial y con poca trayectoria en la semipresencial, facilitadores en el Doctorado en Ciencias de la Educación; y, (2) docentes de Maestría en Educación con cualquier perfil, por considerar que ellos son los formadores en Educación en la modalidad semipresencial para contrastar la experiencia en docencia universitaria. Asimismo, se eligieron 2 profesores expertos de la Universidad Nacional Experimental del Táchira UNET con amplia trayectoria en educación virtual, y experiencia en diseño instruccional para cursos en línea, en el manejo del E-Learning, mediante campus virtuales y hasta redes sociales, cuyas experiencias aportaron elementos estructurales en la construcción de las competencias pedagógicas y tecnológicas del docente virtual UBA.

La recolección de la información ameritó el uso de la técnica de observación directa o participante, desarrollada en las aulas de clase de postgrado de la UBA, registrando cada uno de los datos y eventos tal y como fueron ocurriendo, sin alteraciones y sin establecer comunicación con los sujetos involucrados de manera sistemática. Flores \& Tobón (2003) exponen que el 
trabajo de observación, "requiere, en pos de su validez científica, mucha dedicación al registro riguroso y sistemático y a la redacción de los hechos” (p. 113). En este sentido, se realizó en primer lugar, un sondeo de opinión con los docentes para justificar el planteamiento del problema, posteriormente se recurrió a la observación participante de las clases presenciales al inicio de los programas y el seguimiento a la poca virtualidad que para el momento realizaba el docente.

En segundo lugar, se aplicó la entrevista estructurada en profundidad a los fines de corroborar las conductas observadas frente a la práctica docente en la virtualidad. Mediante esta técnica se buscaba hablar con el informante para poder abordar como percibía la realidad del objeto de estudio relacionado con las competencias pedagógicas y tecnológicas que poseen los docentes de postgrado. Asimismo, se indagó acerca de su desempeño desde su práctica virtual. En la aplicación de las entrevistas se utilizó un guion de entrevista estructurado con aspectos descritos en el cuadro de categorías preestablecidas (cuadro 1). Durante este proceso se utilizó una grabadora para registrar y captar detalles del diálogo, sobre las experiencias, situaciones y puntos de vista de los actores sociales.

Cuadro 1. Categorías apriorísticas

\begin{tabular}{|c|c|c|c|}
\hline CATEGORIAS & SUBCATEGORIAS & DIMENSIONES & TECNICAS \\
\hline Educación Virtual & Acción docente & $\begin{array}{l}\text { 1. Diseño de la práctica. } \\
\text { 2. Proporcionar herramientas y } \\
\text { recursos } \\
\text { 3. Facilitador de Aprendizaje } \\
\text { constructivista } \\
\text { 4. Garantizar calidad del aprendizaje } \\
\text { en línea. }\end{array}$ & $\begin{array}{l}\text { Entrevista en } \\
\text { profundidad }\end{array}$ \\
\hline \multirow[b]{2}{*}{ Competencias } & $\begin{array}{l}\text { Competencias } \\
\text { Pedagógicas }\end{array}$ & $\begin{array}{l}\text { 1. Saber del docente } \\
\text { 2. Práctica docente } \\
\text { 3.Enseñanza Virtual }\end{array}$ & \multirow[b]{2}{*}{$\begin{array}{l}\text { Entrevista en } \\
\text { profundidad }\end{array}$} \\
\hline & $\begin{array}{l}\text { Competencias } \\
\text { Tecnológicas }\end{array}$ & $\begin{array}{l}\text { 1. Comunicativa } \\
\text { 2. Pedagógica } \\
\text { 3. De gestión } \\
\text { 4. Investigativa } \\
\end{array}$ & \\
\hline \multirow{2}{*}{$\begin{array}{l}\text { Diseño } \\
\text { Instruccional }\end{array}$} & Sustento Teórico & $\begin{array}{l}\text { 1. Teorías descriptivas y prescriptivas. } \\
\text { 2.Teorías didácticas y de aprendizaje }\end{array}$ & \multirow{2}{*}{ Revisión Bibliográfica } \\
\hline & Modelos & $\begin{array}{l}\text { 1. García Aretio } \\
\text { 2. ADDIE }\end{array}$ & \\
\hline $\begin{array}{c}\text { Docente } \\
\text { Universitario }\end{array}$ & Desempeño & $\begin{array}{l}\text { 1. Saber } \\
\text { 2. Saber hacer } \\
\text { 3. Saber ser }\end{array}$ & $\begin{array}{l}\text { Entrevista en } \\
\text { profundidad }\end{array}$ \\
\hline
\end{tabular}

Fuente: Buitrago (2018)

En relación con la fiabilidad o credibilidad de la investigación, la fiabilidad externa, según Martínez (2007), al estudiar una realidad en cualquier momento o en situaciones diferentes, los investigadores independientes llegan a los mismos resultados, confirmada con los hallazgos de las investigaciones que son antecedentes de la investigación donde se contrastan resultados comunes. La fiabilidad interna según el autor, al estudiar la misma realidad concuerdan en sus conclusiones; varios observadores como el caso de los expertos en Diseño Instruccional, a quienes se aplicó otra entrevista en profundidad especificando categorías apriorísticas derivadas del objetivo de la investigación, llegaron a aspectos conclusivos comunes. 


\section{Resultados y Discusión}

El proceso investigativo arrojó desde las opiniones de los actores principales y la percepción de las investigadoras, que el saber está integrado por una serie de competencias relacionadas con el conocimiento que debe tener el docente para poder facilitar su proceso didáctico. Allí se resalta entre otras competencias: que el docente debe ser un pedagogo constructivista por excelencia, es decir responsable de sus propios constructos, que conozca las teorías pedagógicas, los principios de la educación virtual y conocimientos sobre las TIC. Por último, dejan claro que el docente debe analizar las necesidades de los estudiantes, antes de planificar sus saberes en función de los esenciales: ser, conocer y hacer. La pedagogía constructivista a partir de las ideas de Ortiz (2015) se logra condensar de la siguiente manera:

Desde el constructivismo, se puede pensar en dicho proceso como una interacción dialéctica entre los conocimientos del docente y los del estudiante, que entran en discusión, oposición y diálogo, para llevar a una síntesis productiva y significativa: el aprendizaje. Sin embargo, hay que recordar que éste y la forma en que se realice, aun cuando sean constructivistas, están determinadas por un contexto específico que influye en ambos participantes: docente y estudiantes, debido a sus condiciones biológicas, psicológicas, sociales, económicas, culturales, incluso políticas e históricas (p. 97)

En este marco interpretativo el aprendizaje es eminentemente activo e implica un flujo asimilativo de adentro hacia afuera. El estudiante no se limita a copiar el conocimiento, sino que lo construye a partir de elementos personales, experiencia e ideas previas, para atribuir significado y representarse el nuevo conocimiento con sentido adquirido. Como consecuencia, cambia el papel del docente, que pasa de suministrar conocimientos, a participar en el proceso de construcción junto con el estudiante de un conocimiento compartido o ayudado. Por tanto, los diseños instruccionales que coadyuven a la construcción del conocimiento parten de un modelo que genere nuevos aprendizajes frente a los que ya trae el estudiante, para lo cual el rol del docente o tutor virtual es el de proporcionarle, los materiales y herramientas necesarias.

Para el proceso de análisis de los resultados, se utilizó el programa Atlas ti, como herramienta informática para la aplicación de procesos: análisis, síntesis, y descripción de la información, por ser un análisis inductivo de magnitudes de datos verbales. Este programa, según Martínez (2007) no pretende automatizar el proceso de análisis, sino que sirve de ayuda a las personas interesadas en agilizar muchas de las actividades implicadas en el análisis cualitativo y la interpretación.

El análisis inductivo se desarrolló a través de los procesos de: categorización, estructuración y contrastación, sugeridos por el autor para las investigaciones y estudios acerca de procesos creativos, con la finalidad de relacionar datos e ideas apropiados en la solución del problema. De esta manera, el trabajo estuvo centrado en leer y releer los protocolos de información recabados, para iniciar una previa codificación la cual fue depurando en función de seleccionar aquellos datos pertinentes al objeto de estudio, con el apoyo de tablas de Excel.

Los textos guardados en la unidad hermenéutica del Atlas Ti, fueron divididos en porciones o unidades temáticas, las cuales expresaron una idea o un concepto para ser posteriormente 
codificados. Los códigos y categorías que fueron emergiendo del proceso de categorización, son llamados palabras claves, las cuales, en el enfoque cualitativo, se convierten en ideas, conceptos, interpretaciones y van apareciendo en orden alfabético con dos números entre paréntesis.

La segunda fase del método etnográfico orientó la aplicación de la entrevista en profundidad a los docentes de postgrado de la Universidad Bicentenaria de Aragua (UBA), extensión San Cristóbal y los expertos de la Universidad Nacional Experimental del Táchira (UNET) seleccionados como informantes clave. De igual manera, se realizó la observación directa y participante en las aulas de clase de la UBA. El análisis ofrece las realidades vivenciales de estos actores sociales desde el acto educativo semipresencial, en lo específico en sus competencias como docentes virtuales, expresado desde las categorías apriorísticas para la generación de los elementos estructurales que constituyeron las nuevas categorías que emergen, para la construcción de las competencias pedagógicas y tecnológicas que debe poseer el docente virtual.

Para dicho análisis, considerada la evidencia empírica, se aplicó el proceso de inducción analítica, donde se empleó la codificación axial o abierta, a través del análisis asistido por el software Atlas ti. El proceso llevado a cabo facilitó la reducción de la información con la intención de agrupar los datos primarios para la codificación que se expresa en categorías. Los mismos son producto de la operación concreta por la que se asigna a cada fragmento del texto del documento primario, un indicativo propio de las categorías apriorísticas antes descritas.

Cuadro 2. Categorías Emergentes

\begin{tabular}{|c|c|c|}
\hline Dimensiones & Subcategorías & Categorías \\
\hline Facilitador de Aprendizajes & \multirow{9}{*}{ Acción Docente } & \multirow{15}{*}{ Docente Virtual } \\
\hline Capacitación Docente & & \\
\hline Practica Virtual & & \\
\hline Conocimiento Tecnológico & & \\
\hline Práctica Tradicional & & \\
\hline Diseño de la práctica & & \\
\hline Manejo de herramientas y recursos & & \\
\hline Pedagogo Constructivista & & \\
\hline Calidad del Aprendizaje & & \\
\hline Diseño de la Instrucción & \multirow{2}{*}{ Diseño Instruccional } & \\
\hline Sustento Teórico & & \\
\hline Capacitación Docente & \multirow{2}{*}{ Formación Docente } & \\
\hline Diseño de la Instrucción & & \\
\hline Funciones del Docente & \multirow{2}{*}{$\begin{array}{l}\text { Postulados de la } \\
\text { UNESCO }\end{array}$} & \\
\hline Gestión del Conocimiento & & \\
\hline Saber hacer del docente & \multirow{2}{*}{$\begin{array}{l}\text { Competencias } \\
\text { Tecnológicas }\end{array}$} & \multirow{3}{*}{ Competencias } \\
\hline Infraestructura Tecnológica & & \\
\hline Saber del Docente & $\begin{array}{l}\text { Competencias } \\
\text { Pedagógicas }\end{array}$ & \\
\hline
\end{tabular}

Fuente: Buitrago (2018) 
El análisis descriptivo, reflejó en forma detallada un sistema de categorías emergentes (cuadro. 2) obtenido del proceso de codificación y categorización donde se exhiben: Códigos, Dimensiones y Subcategorías que manifiestan la esencia de la realidad estudiada y se constituyen en elementos fundamentales para la consecución del objetivo.

La tercera fase del método etnográfico relacionada con el nivel de objetividad de la investigación estuvo definida por varios aspectos, los cuales al decir de Martínez (2007) estuvieron relacionados con una cuidadosa selección de la muestra intencional, la empatía que se logró en los sujetos a investigar y su buen nivel de confiabilidad y validez. De allí que, al considerar el antepuesto análisis descriptivo de la información recabada mediante las tres fuentes (docentes, expertos e investigadoras) se consideraron la acciones y respuestas de los hechos estudiados para poder determinar el qué, cómo, quién y dónde se desarrollaron los hechos, por lo cual, operó tanto la percepción como la subjetividad de las investigadoras.

El proceso de control cruzado de la información desde las entrevistas a docentes, entrevistas a expertos, observación directa y los planteamientos teóricos, brindó amplitud, consistencia y relevancia al fenómeno estudiado; contar con percepciones coincidentes y opuestas en relación con el objeto de estudio, lo cual permitió descubrir los elementos estructurales que vienen a dar inicio a la consolidación de los constructos como sustento y fundamento para la construcción de competencias pedagógicas y tecnológicas en los procesos de diseño instruccional.

Los hallazgos encontrados para la categoría: Docente Virtual reflejó algunas coincidencias al triangular las fuentes de información; según los docentes: Prevalece la práctica docente tradicional, por lo que manifiestan, falta de experiencia para práctica virtual y desconocimiento de estrategias. Es decir, son inmigrantes digitales, lo que conduce a una inminente necesidad de capacitación de los docentes en los procesos de diseño instruccional. Estos elementos se convirtieron en premisas para la construcción de competencias pedagógicas y tecnológicas del docente virtual de la UBA, dado que son los mismos actores principales del acto pedagógico quienes dejan ver sus falencias y sus necesidades de formarse en el área.

Por su parte, los expertos frente a la dimensión Funciones del Docente, han dicho: El docente en la educación virtual es un docente que debe conocer, manejar las TIC, el saber pedagógico y la integración y aplicación de estos saberes en el desarrollo de procesos educativos virtuales. Adicional a lo anterior debe ser un experto en los contenidos teóricos de su especialidad. Es decir, debe darse la actualización tecnológica y el docente ser diseñador de las condiciones del aprendizaje, así como, tener habilidades y destrezas en su saber docente. En suma, han señalado la necesidad de formación del docente virtual UBA. De manera que, si el experto en el área de diseño instruccional coincide con tales necesidades, reafirma la intención de las investigadoras, quienes dejan claro que: Se aprecia nulo el diseño de la guía didáctica del docente para la virtualidad y observa que no utilizan el aula virtual lo cual delimita el uso de recursos y herramientas virtuales. Allí tampoco se observa formación continua del docente, pero si sus inclinaciones por capacitarse como tutor virtual.

En atención a lo anterior, se justifica la necesidad de potenciar la acción docente como facilitador del aprendizaje. En primer lugar, a su vez es una oportunidad de aprendizaje, pero en la práctica 
se está en una transición de lo tradicional a lo virtual, por lo que impera la insuficiente capacitación docente quien no utiliza el aula virtual, lo cual se hace indispensable para involucrar al estudiante en la construcción de sus aprendizajes. Es decir, la falta de experiencia para la práctica virtual conduce a una necesidad de actualización tecnológica ya que se observa el no uso recursos y herramientas necesarios para potenciar la actividad docente desde la virtualidad.

En segundo lugar, en la categoría Competencias referidas al ser, hacer, conocer y a convivir como habilidad y destreza del docente, relacionadas con las subcategorías Competencias Tecnológicas y Pedagógicas, debe estar en comunión con sus competencias genéricas y específicas, las que, al ser consultadas con la realidad, surge la necesidad de querer indagar sobre los recursos para la adquisición de estas. Así desde el escenario estudiado se observa la limitación que existe en el proporcionar herramientas online, tal situación es precisamente por las habilidades y destrezas que carece el docente virtual, las cuales están dadas por su preparación académica, centrada en lo tradicional. Es por ello, que desde la presente investigación, se busca consolidar el hacer del docente y fortalecer el saber desde su formación inicial.

En consecuencia, surgen de la presente investigación los siguientes aspectos trascendentales tales como: Los docentes deducen que las competencias tecnológicas se traducen en el Saber hacer del docente, y dejan ver necesidad de capacitación como docente virtual, lo cual conduce a la formación del docente en estas competencias, por cuanto viene de una formación pedagógica inicial insuficiente para dotar al docente de todo el conocimiento y las destrezas que necesitarán a la hora de enfrentarse a la Educación Virtual, por lo que requieren de una formación continua.

Por su parte los expertos, concuerdan en que se requiere de docentes con competencias pedagógicas y tecnológicas que, desde el saber del docente, conduzcan al aprender a aprender, aprender a hacer, aprender a convivir y aprender a ser, sustentados en los objetivos de la UNESCO. Asimismo, se observó la falta de formatos preestablecidos para el diseño de la instrucción y consideran que requieren formación en competencias pedagógicas y tecnológicas del docente virtual. En este sentido, la capacitación que aluden necesitar los docentes se refleja en la falta de estrategias para diseñar la instrucción en ambientes virtuales.

En consecuencia de lo expuesto, desde esos aspectos coincidentes resulta que el aprendizaje virtual debe orientarse desde la perspectiva constructivista e interactiva y de calidad para orientar el mismo. Este a su vez deberá ser: colaborativo, cooperativo, compartido, autónomo, continuo y estratégico. Asimismo, el aprendizaje desde la modalidad virtual deberá interrelacionar dimensiones como el saber (conocimiento), el saber hacer (habilidades y destrezas) y el saber ser (afecto), las cuales estuvieron presentes en los elementos estructurales que conforman la construcción de las nuevas competencias del docente virtual.

\section{Competencias pedagógicas y tecnológicas en el contexto de estudio}

A partir de los resultados del presente estudio, surgen elementos estructurales que conducen a la creación de competencias pedagógicas y tecnológicas para los docentes virtuales de la UBA, como el contexto donde surge la necesidad. Estos elementos están dados por: el saber hacer del docente y la infraestructura tecnológica, propios de las competencias tecnológicas, por una parte, y por la otra, el saber del docente, propio de las competencias pedagógicas 
Las competencias requeridas para los procesos de Diseño Instruccional en la Educación Virtual de la UBA, se sustentan en conocimientos, actitudes, habilidades y destrezas expresadas en el ser, conocer, hacer y convivir del docente para orientar su práctica virtual, mediante un diseño de la instrucción que se fundamente en teorías relativas al aprendizaje humano, a enfoques tecnológicos para conducir al estudiante a desenvolverse en diferentes contextos desde una mediación pedagógica que lo llevará a probarse, evaluarse y revisarse de forma continua, atendiendo de forma efectiva las necesidades de los estudiantes.

\section{Competencias Pedagógicas}

El conocimiento y la actitud que tiene el docente para orientar su práctica pedagógica desde su formación inicial. Es decir, cuál es su perfil y que es lo que sabe a partir de su vocación, comprensión, su disposición cognitiva, y socioafectivas para lograr una convivencia con sus estudiantes, salvando alcances y limitaciones (ver cuadro 3).

Cuadro 3. Elementos estructurales emergentes para las Competencias Pedagógicas del Docente Virtual

\begin{tabular}{|c|c|c|}
\hline Elementos & Competencias & Dimensión \\
\hline \multirow[t]{3}{*}{ Saber del docente } & $\begin{array}{l}\text { - Formación inicial como docente con capacitación y formación } \\
\text { - } \quad \text { Continua en TIC. } \\
\text { - } \quad \text { Define las competencias: saberes esenciales: ser, conocer y hacer; y, } \\
\text { los criterios de desempeño en las actividades de aprendizaje } \\
\text { - conoce las metodologías de investigación propias de la ciencia que } \\
\text { enseña } \\
\text { - Potencia el aprendizaje en los educandos. } \\
\text { Utiliza las técnicas adecuadas, redactar artículos sobre las } \\
\text { investigaciones que realiza el estudiante }\end{array}$ & Conocer \\
\hline & $\begin{array}{l}\text { - } \text { Fomenta sus habilidades en pro de las metas trazadas } \\
\text { - Implementa estrategias didácticas y el uso de las Tecnologías. } \\
\text { - Mediador pedagógico en el entorno virtual } \\
\text { - } \quad \text { Planifica procesos didácticos sobre: aprendizaje colaborativo, } \\
\text { cooperativo, compartido, autónomo, aprendizaje continuo y } \\
\text { aprendizaje estratégico } \\
\text { - Pedagogo constructivista por excelencia. } \\
\text { - Habilidad y disposición para aprender a utilizar diferentes } \\
\text { herramientas virtuales }\end{array}$ & Hacer \\
\hline & $\begin{array}{l}\text { - Realiza proyectos en colaboración entre instituciones y grupos de } \\
\text { investigación. } \\
\text { - Apoya a sus estudiantes y dirige las investigaciones, con el fin de } \\
\text { difundir el conocimiento producido. }\end{array}$ & Convivir \\
\hline $\begin{array}{l}\text { Orientación de la } \\
\text { enseñanza }\end{array}$ & $\begin{array}{l}\text { - Orienta la enseñanza, sobre todo el manejo de herramientas para } \\
\text { investigar en la web } \\
\text { - } \quad \text { Promueve la investigación en el estudiante. } \\
\text { - Emplea la observación y el registro de su experiencia docente para } \\
\text { - } \quad \text { Se capacita como docente virtual }\end{array}$ & Ser \\
\hline $\begin{array}{l}\text { Vocación docente } \\
\text { y humanista }\end{array}$ & $\begin{array}{l}\text { - Tiene vocación y aplica estrategias de motivación en la virtualidad } \\
\text { - } \quad \text { Afianzarse en la teoría humanista para fortalecer el perfil profesional }\end{array}$ & Convivir \\
\hline
\end{tabular}

Fuente: Buitrago (2018) 


\section{Competencias Tecnológicas}

Los conocimientos habilidades y destrezas que posee el docente para saber hacer su práctica online, siempre que cuente con una infraestructura tecnológica que le permita el uso de estrategias didácticas mediante el manejo y uso adecuado de herramientas y recursos para diseñar cursos en línea y desempeñarse como docente virtual (ver cuadro 4).

Cuadro 4. Elementos estructurales emergentes para las Competencias Tecnológicas del docente virtual

\begin{tabular}{|c|c|c|}
\hline Elementos & Competencias & Dimensión \\
\hline \multirow[b]{2}{*}{$\begin{array}{l}\text { Saber hacer } \\
\text { del } \\
\text { docente }\end{array}$} & $\begin{array}{l}\text { - Usa recursos virtuales para comunicarse con estudiantes. } \\
\text { - } \quad \text { Usa herramientas virtuales para preparar su práctica } \\
\text { - Diseña cursos virtuales }\end{array}$ & Hacer \\
\hline & $\begin{array}{ll}\text { - } & \text { Tiene aptitudes, habilidades y destrezas para preparar su } \\
& \text { práctica virtual } \\
\text { - } & \text { Tiene experiencia en docencia virtual. } \\
\text { - } & \text { Conoce metodología interactiva. } \\
\text { - } \quad \text { Conoce la tecnología }\end{array}$ & Conocer \\
\hline \multirow{2}{*}{$\begin{array}{l}\text { Infraestructura } \\
\text { Tecnológica }\end{array}$} & $\begin{array}{ll}\text { - } & \text { Conoce beneficios del aprendizaje en línea. } \\
\text { - } & \text { Conoce estrategias de docencia virtual } \\
\text { - } & \text { Conoce Metodologías de aprendizaje. } \\
\end{array}$ & Conocer \\
\hline & $\begin{array}{l}\text { - Maneja equipos para apoyar su práctica virtual } \\
\text { - } \quad \text { Diseña en plataforma tecnológica- } \\
\text { - } \quad \text { Manejo de equipos para apoyar su práctica virtual }\end{array}$ & Hacer \\
\hline
\end{tabular}

Fuente: Buitrago (2018)

Los elementos estructurales para las competencias pedagógicas y tecnológicas surgen como resultado del análisis inductivo de las diferentes categorías y subcategorías emergentes atendiendo tanto a la información recabada de los informantes clave, la literatura especializada para sustentar los referentes teóricos de la investigación (UNESCO-IIPE, 2018; MEN, 2013; Observatorio de Innovación Educativa, 2015), así como la percepción de las investigadoras en la triangulación de las fuentes de información. Es así que, a continuación se presenta la realidad descubierta para la creación de competencias pedagógicas y tecnológicas del docente virtual de la UBA, en el proceso de diseño instruccional, que conduce a las siguientes premisas:

a) las competencias tecnológicas, conocidas como técnicas se relacionan con el aprender a hacer, es decir, lo relacionado con lo práctico, técnico y científico de la enseñanza y aprendizaje en la virtualidad;

b) las competencias pedagógicas, conocidas como cognitivas y formativas están representadas en el aprender a conocer, a comprender, a ser y a convivir, todo relacionado con valores, así como en las actitudes profesionales, sociales, filosóficas, necesarias en el arte de enseñar en la modalidad virtual.

\section{Conclusiones}

Las diversas situaciones que enfrenta el docente virtual llevan al éxito o al fracaso en una modalidad de la enseñanza experimentada en las instituciones universitarias, en este caso en el 
área de postgrado de la UBA, direcciona acciones para la formulación de competencias pedagógicas y tecnológicas innovadoras de diseño, con el fin crear estándares que conduzcan a perfeccionar los procesos de enseñanza y aprendizaje. El proceso investigativo permitió dar respuesta a los objetivos de la investigación de la siguiente manera,

a) Al identificar las competencias pedagógicas del docente virtual de la UBA, se aprecia una formación inicial lejos de la virtualidad, ya que los docentes provienen de áreas del conocimiento propias de sus profesiones de ingeniero, abogados, educadores con postgrados en planificación, educación, entre otras, dejando ver su saber cómo docente, junto con la necesidad de formación continua. La misma se acrecienta en tiempos de pandemia donde la nueva realidad conduce a una educación desde la virtualidad con docentes preparados desde el saber, saber hacer, conocer y convivir con la tecnología educativa.

b) Al diagnosticar las competencias tecnológicas del docente virtual de la UBA, se estima una escasa habilidad y destreza en el manejo de herramientas y recursos por parte de los docentes, así como el poco apoyo institucional con una infraestructura tecnológica que los conduzca a una verdadera práctica virtual, por ello, reiteran en varias oportunidades la necesidad de capacitación, la cual se evidencia en la actualidad donde la educación virtual se traduce en el modelo educativo que regirá las futuras generaciones.

c) Para determinar el proceso de Diseño Instruccional en la educación virtual se contempla un proceso que conlleva diferentes pasos dependiendo del modelo; el docente, quien debe diseñar la instrucción que conduzca a la educación virtual de calidad. Para ello, deberá seleccionar el modelo o crear el propio, siempre que se adapte a las necesidades de sus participantes y sustentado en teorías de soporte del diseño. En este sentido, el apoyo teórico de las competencias está claramente fundamentado en el estudio desde las teorías de la enseñanza y el aprendizaje y enfoques tecnológicos.

\section{Referencias}

Aliendres, N. (2012). Competencias del docente para el logro de una educación de calidad en el Instituto Universitario de Tecnología de Puerto Cabello [Trabajo de Maestría, Universidad de Carabobo]. http://www.grupocieg.org/archivos/Competencias\%20docentes\%20Nidia\%20Aliendres\%20UC.pdf

Buitrago, B. (2018). Competencias pedagógicas y tecnológicas de los docentes universitarios en los procesos de diseño instruccional para la educación virtual [Trabajo de Maestría no publicado]. Universidad Nacional Experimental del Táchira.

Carrillo, M. \& Roa, L. (2019). Diseñando el aprendizaje desde el modelo ADDIE [Trabajo de Especialización, Universidad de la Sabana]. Repositorio institucional - Universidad de La Sabana.. https://intellectum.unisabana.edu.co/handle/10818/35378

Calderón, M., A. (2020). Elementos clave de la virtualidad en la educación superior. Revista Electrónica Calidad En La Educación Superior, 11(2), 80 - 104. https://doi.org/10.22458/caes.v11i2.3322

Cohen-Granados, J., Linares-Morales, J., \& Briceño-Ariza, L. (2020). Caracterización de la cultura innovativa en la cooperación universidad-empresa. IPSA Scientia, Revista científica Multidisciplinaria, 5(1), 46-53. https://doi.org/10.25214/27114406.963 
De-Vincenzi, A., Marcano, D., \& Macri, A. (2020). La práctica educativa bajo el lente de la teoría de la actividad. IPSA Scientia, Revista científica Multidisciplinaria, 5(1), 159-176. https://doi.org/10.25214/27114406.1033

Díaz, A. \& Serra, L. (2020). Competencias digitales del docente universitario. SUMMA. Revista disciplinaria en

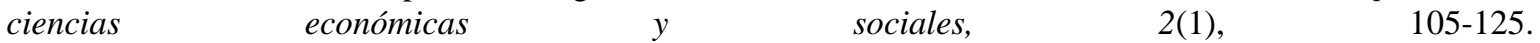
https://aunarcali.edu.co/revistas/index.php/RDCES/article/view/113

Flores, R. \& Tobón, A. (2003). Investigación Educativa y Pedagógica. McGraw-Hill/interamericana, S. A. H.

Gamarra, F. (2021). M-Learning una oportunidad para el sistema educativo. Polo del Conocimiento, 6(1), 998-1019. https://polodelconocimiento.com/ojs/index.php/es/article/view/2195

García Aretio, L. (14 de abril de 2020). Una Unidad Didáctica, un Tema, una Lección..., en formato digital. Contextos universitarios mediados. https://aretio.hypotheses.org/1933

Jiménez Puello, J. de J. (2015). Estudio sobre los estándares TIC en educación en los futuros docentes de la Facultad de Educación de la Universidad Complutense de Madrid. [Tesis Doctoral], Universidad Complutense de Madrid]. E-Prints Complutense. https://eprints.ucm.es/id/eprint/30925/

Martínez, M. (2007), Ciencia y Arte en la Metodología Cualitativa. Métodos Hermenéuticos, Métodos Fenomenológicos. Métodos Etnográficos (2da. Edición). Editorial Trillas.

Ministerio de Educación Nacional de Colombia (MEN-2013). Competencias TIC para el Desarrollo Profesional Docente. Colección Sistema Nacional de Innovación Educativa con uso de Nuevas Tecnologías. http://www.premiosantillana.com.co/pdf/competencias tic.pdf

Observatorio de Innovación Educativa (2015). Edu Trends. Radar de Innovación Educativa. Tecnológico de Monterrey. http://observatorio.itesm.mx/edutrendsradar2015/

Ortiz Granja, D. (2015). El constructivismo como teoría y método de enseñanza. Sophia, Colección de Filosofía de la Educación, (19), 93-110. https://www.redalyc.org/articulo.oa?id=441846096005

Prada, L. (2010).Modelo Andragógico basado en competencias TIC para docentes universitarios, un preámbulo hacia la ciberdidaxia [Tesis doctoral no publicada]. Universidad Interamericana de Educación a Distancia de Panamá.

Roblizo Colmenero, M. \& Cózar Gutiérrez, R. (2015). Usos y competencias en TIC en los futuros maestros de educación infantil y primaria: hacia una alfabetización tecnológica real para docentes. Pixel-Bit. Revista de Medios y Educación, (47). 23-39. http://www.redalyc.org/articulo.oa?id=36841180002

Russi, N., (2020). El desafío de la educación virtual en un contexto de pandemia. Red Sociales, Revista del Departamento de Ciencias Sociales, 7(5). 19-24. http://www.redsocialesunlu.net/wpcontent/uploads/2020/09/3.-El-desaf\%C3\%ADo-de-la-educaci\%C3\%B3n-virtual-en-un-contexto-depandemia..pdf

Sánchez, A. (2020). Aprendiendo desde la virtualidad. Competencias en contextos educativos virtuales. Revista Educ@ción en $\quad$ Contexto, $\quad$ VII(13). 9-32. https://educacionencontexto.net/journal/index.php/una/article/view/145/265

Siemens, G. (2010). Conociendo el conocimiento (E. Quintana, D. Vidal, L. Torres \& V. Castrillejo, Trads.). Nodos ELE. (Obra orifinal publicada en 2006). http://davidal.es/wpcontent/uploads/2020/09/Siemens.Conociendoelconocimiento.pdf

UNESCO - IIPE (2018). Planificar la Educación, construir el futuro. $10^{a}$ Estrategia de Medio término 2018-2021. Instituto Internacional de Planeamiento de Educación.. https://www.buenosaires.iiep.unesco.org/sites/default/files/archivos/10\%C2\%BAEMT.pdf 